\title{
Extraction of feature values using protein molecular surface data and prediction of significant spot for preferential binding of ligands
}

\author{
Aoi Fukazawa $a^{\mathrm{a},{ }^{*},}$ Ryota Tamoria ${ }^{\mathrm{a}}$, Ryo Nishide ${ }^{\mathrm{b}}$, Takenao Ohkawa ${ }^{\mathrm{a}}$ \\ ${ }^{a}$ Graduate School of System Informatics Kobe University 1-1, Rokkodai, Nada, Kobe 657-8501 JAPAN \\ ${ }^{b}$ The Center for Data Science Education and Research, Shiga University, 1-1-1 Banba, Hikone, Shiga 522-8522, Japan \\ *aoi@cs25.scitec.kobe-u.ac.jp
}

\begin{abstract}
Proteins often bind to other compounds at the concave site which is referred to as a pocket, and it is known that the binding site is locally similar with proteins binding to the same compound. In this paper, we propose a new method to predict the important structures when binding to the ligand in the pocket (described as "significant spot"), by focusing on the distance between the surfaces of proteins. Experiments were conducted with 60 kinds of proteins to extract significant spot for each protein. As a result, significant spot were extracted with high accuracy, and the effectiveness of the proposed method was confirmed.
\end{abstract}

Keywords: protein, structure, extraction.

\section{Introduction}

Most proteins express a variety of functions by interacting with low molecular compounds (ligands). ${ }^{(1,2)}$ This interaction often occurs in hollows of molecular surface (pockets), and these sites are known to be locally similar among the proteins binding to similar ligands. It is time and cost consuming to analyze the properties of proteins and interacting ligands by scientific experiments, so analysis is performed using computers. ${ }^{(3-7)}$

In this paper, we propose a method to predict the important structure when binding to the ligand in the pocket (described as "significant spot" from here on). This significant spot could be extracted as a similar local molecular surface binding to the similar ligand. Therefore, in the proposed method, the molecular surface is expressed as $3 \mathrm{D}$ point cloud data consisting of characteristic points (feature points), and the set of feature points similar to a certain pocket is extracted from a large number of proteins. Then, based on the set of obtained feature points, biclustering is performed to find a set of local feature points common to many proteins as the significant spot. When evaluating the similarity of a set of feature points, a feature value is used. The feature value is created by setting the neighborhood area based on the distance from the feature point in the $3 \mathrm{D}$ space, then referring to the neighboring data points for each area. In the proposed method, three types of feature value are introduced. They reflect the positional and physical properties around feature points, which enable to compare more detailed information about pockets.

\section{Extraction of feature value using molecular surface data and prediction of important binding sites}

\subsection{Extraction of protein pocket}

In the proposed method, efvet data acquired from the database eF-site $^{(8)}$ is used as $3 \mathrm{D}$ point cloud data that indicates the surface properties of the protein. This efvet data is given physical property values such as electrostatic potential and hydrophobicity. It describes the nature of molecular surface calculated with reference to the atomic data of the public database Protein Data Bank (PDB) ${ }^{(9)}$, which contains information about the structures of proteins. The molecular surface is represented by polygons in efvet, but it is not efficient to compare the point cloud data added with chemical properties to each vertex of polygon because the computational cost is high. Moreover, it is unlikely that the sites which bind to the same ligand will be completely identical, including the surrounding sites. Thus, we must 


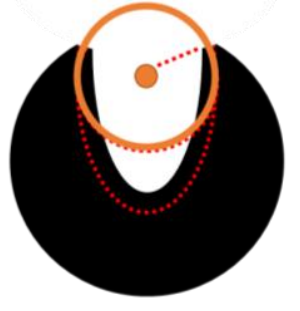

a. The method of extracting a range $b$. from the center of the pocket

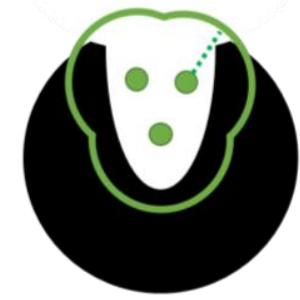

the method of using position of the ligand
Fig. 1. Pockets of protein.

compare the structural information around polygon data points and evaluate the similarity. Therefore, in order to reduce the data points for comparison, feature points are extracted from polygon data points on the molecular surface of the pocket by using the extraction method ${ }^{(10)}$ based on the maximum and minimum curvatures of the approximate spherical surface. At this time, only the feature points in the pocket are extracted for comparison. Based on the positional information on the binding state of ligands binding to each protein, feature points within a certain distance from the ligand are extracted as points in the pocket. Positional information on the binding state of ligands binding to proteins can be obtained from PDB. Fig. 1 describes two method for a model in which the pocket is elongated. The range of the pocket is expressed in a colored line. The method of extracting a range from the center of the pocket is on the left, and the method of using position of the ligand is on the right. In the left figure, the range is determined by the sphere from the center coordinates of the pocket, so if such a pocket is elongated or has a complicated shape, there is an unextracted area. On the other hand, in the right figure, a pocket can be extracted appropriately, because an area within a certain distance from each atom of the ligand is extracted. In the proposed method, the method using the position of ligand as exemplified on the right figure is adopted.

\subsection{Feature value given to feature point}

The following three feature values are introduced to compare (match) feature points.

- Angle histogram

- Height feature vector

- Physical property histogram

The feature vectors obtained by these three features are normalized and used as input for matching process. Each feature value is described below.

\subsubsection{Angle histogram}
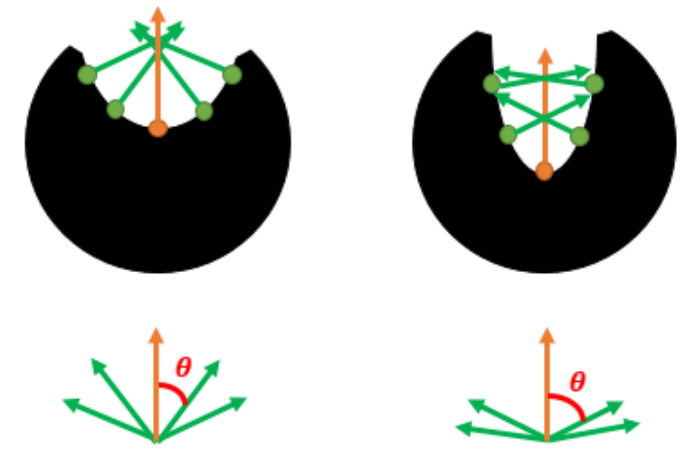

Fig. 2. Protein model and normal vector

The normal vector shows the outer perpendicular direction of the protein at each data point. The angle between normal vectors shows how much the surface changes between the nearby point and the feature point. The larger the angle between the two vectors, the greater the curvature of the surface around the feature point. The smaller the angle, the less change of the surface as it becomes closer to a plane. By separating the nearby points according to the distance from the feature point and creating a histogram, it is possible to capture the shape around the feature point for each distance. This histogram is referred to here as the "angle histogram". This feature value can be obtained by the normal vector of the feature point and the nearby point. Assuming that the normal vector of the feature point is $\overrightarrow{n_{a}}=\left(n_{a 1}, n_{a 2}, n_{a 3}\right)$ and the normal vector of the nearby point is $\overrightarrow{n_{b}}=\left(n_{b 1}, n_{b 2}, n_{b 3}\right)$, the angle $\theta$ between the two normal vectors can be obtained by the following equation.

$$
\theta=\arccos \frac{n_{a 1} n_{b 1}+n_{a 2} n_{b 2}+n_{a 3} n_{b 3}}{\sqrt{n_{a 1}^{2}+n_{a 2}^{2}+n_{a 3}^{2}} \sqrt{n_{b 1}^{2}+n_{b 2}^{2}+n_{b 3}^{2}}}
$$

The $\theta$ obtained by this equation always has a value of 0 to 180 . In the proposed method, we separate the angle $\theta$ into $m$ steps in a constant degree, and $l$ steps based on the distance from the feature point. Thus, a total of $m \times l$ histograms is created. In order to designate this concept of feature value, Fig. 2 shows an example of reproducing a protein surface and normal vectors. (In the figure, a three-dimensional normal vector is shown as a two-dimensional vector for convenience.) The bottom part of the figure shows only the normal vectors of each pocket. The orange point is extracted as the feature point, and the angle between the normal vector of the orange point and the normal vector of the nearby green point is found. These two surfaces are seemingly similar pockets, but they can be separated by this feature value.

On the other hand, Fig. 3 shows an example of reproducing a protein surface with a similar histogram. 

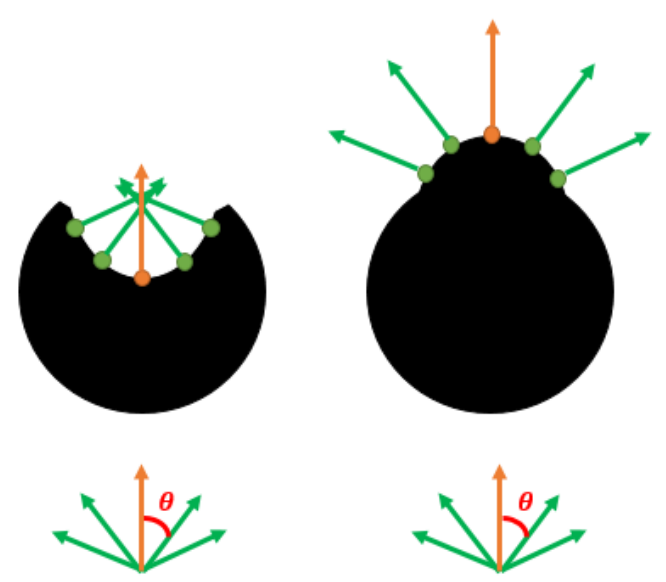

Fig. 3. Protein model with similar angle histograms.

These two surfaces create histograms with similar distributions, using this feature value that focuses only on the angle between the normal vectors. However, even though these two figures should be clearly distinguished, they cannot be separated by this feature value itself. Therefore, in addition to this feature value, the distance of each data point from the contact surface of the feature point is used as the feature value that represents the positional feature.

\subsubsection{Height feature vector}

In angle histograms, similar histograms may be generated regardless of whether the curvature near the feature points is positive or negative.

Therefore, the height of each data point viewed from the contact surface of the feature point is introduced as the feature value. The contact surface of the feature point can be calculated from the normal vector. The position of the nearby point viewed from the feature point is calculated based on the contact surface of the feature point. By separating these values by the distance between the feature point and the data point and creating feature vectors, it is possible to capture the distribution of elevations at each distance viewed from the feature point. In this method, this feature vector is called "height feature vector". When the normal vector $\overrightarrow{n_{a}}=\left(n_{a 1}, n_{a 2}, n_{a 3}\right)$ of the feature point, the coordinate $\vec{a}=\left(a_{1}, a_{2}, a_{3}\right)$ of the feature point, and the coordinate $\vec{b}=\left(b_{1}, b_{2}, b_{3}\right)$ of the nearby point are assigned, then the height $h$ of the nearby point viewed from the contact surface of the future point can be calculated by the following formula.

$h=\frac{n_{a 1}\left(b_{1}-a_{1}\right)+n_{a 2}\left(b_{2}-a_{2}\right)+n_{a 3}\left(b_{3}-a_{3}\right)}{\sqrt{n_{a 1}^{2}+n_{a 2}^{2}+n_{a 3}^{2}}}$

The larger the value of $h$, the more the nearby point is
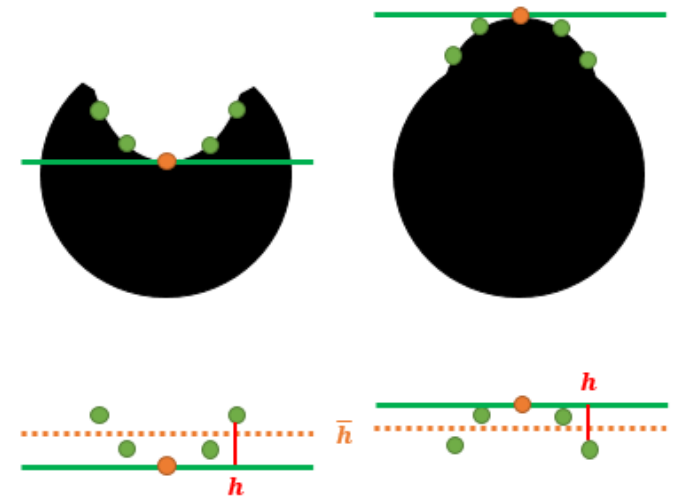

Fig. 4. Protein model and height of feature points from molecular surface.
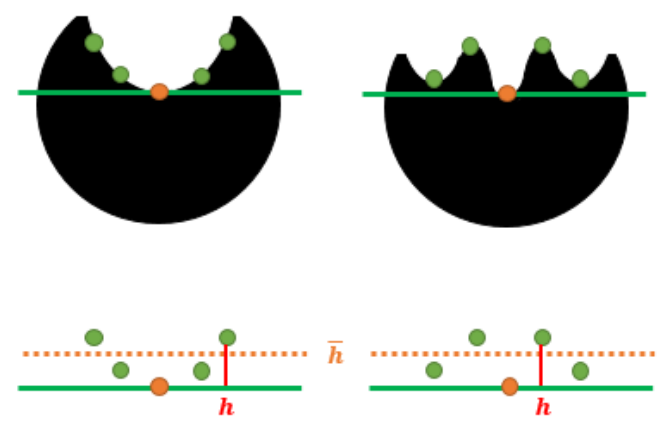

Fig. 5. Protein model with similar height feature vectors.

in a position bulging from the feature point.

In the proposed method, the distance between two points is classified into $l$ levels, and the average $\bar{h}=$ $\frac{1}{m} \sum_{k} h$ of the height $h$ of $k$ nearby points in the region is used as the feature value for each level. This feature value is an $l$-dimensional feature vector, and not a histogram. Therefore, unlike the angle histogram, it is possible to hold minute differences in value.

In order to express the concept of this feature value, Fig. 4 shows an example of reproducing a protein surface, an example of each nearby point, and an image of the average height $\bar{h}$. This feature value holds the average of the elevations of the nearby points with respect to the feature point, which is in the area at a constant distance from the feature point. By using this feature value, it is possible to distinguish the surface of the pocket of the two figures in Fig. 4 that cannot be captured only by the angle histogram introduced in the previous section. On the other hand, Fig. 5 shows a figure reproducing a surface with similar height feature vectors. These two surfaces cannot be distinguished by this feature value, but they can be distinguished by the angle histogram. 


\subsubsection{Physical property histogram}

Information of physical properties can be obtained from efvet mainly on electrostatic or hydrophobic properties. Referring to the electrostatic potential and hydrophobicity values described in efvet, the feature value about the physical property is created. The electrostatic potential values are classified into $c$ stages. Hydrophobicity values are classified into $d$ types based on the amino acid hydrophobicity index ${ }^{(11)}$ proposed by Kyte et al. This creates a histogram. At this time, according to the distance between the feature point and the nearby point, the histogram is divided into $e$ stages, and a total of $c \times d \times$ $e$ dimensional histogram is created. In this paper, this histogram is called "physical property histogram". By this feature value, it is possible to compare the properties for each data points, and thus, more detailed comparison can be performed.

\subsection{Matching process of feature points}

A pocket is represented as a set of feature points to which feature values are given. Here, feature points are compared to evaluate the similarity between pockets. By comparing the coordinates of points and feature value, we search for the point cloud whose feature points are structurally and functionally similar. As a result, with respect to feature points representing two pockets, it is possible to obtain a set of feature point pairs in high speed that can be regarded as similar among the pockets. With a general-purpose CPU, we compare sets of feature points based on feature value and generate a list of similar feature points corresponding to $3 \mathrm{D}$ space ${ }^{(12)}$.

In the proposed method, in the feature point pairs listed as matching candidates based on the positional relationship of feature points, the feature values are compared to determine whether the pairs are similar or not. Here, we define the similarity between feature points to determine if the feature point pairs are similar. Assuming that the similarity for each feature value of a feature point pair is $s(1), s(2), s(3)$, the similarity $S$ is defined by the following equation.

$$
S=1-s(1)-s(2)-s(3)
$$

Assuming that the weights are $w_{1}, w_{2}$ and $w_{3}$, the similarity $s(1)$ of the angle histogram, the similarity $s(2)$ of the height feature vector, and the similarity $s(3)$ of the physical property histogram are defined respectively by the following equations.

$$
\begin{aligned}
& s(1)=\sum_{x=1}^{m * l}\left|P_{1}(x)-Q_{1}(x)\right| \\
& s(2)=\sum_{x=1}^{l}\left|P_{2}^{\prime}(x)-Q_{2}^{\prime}(x)\right| \\
& s(3)=\sum_{x=1}^{c * d * e}\left|P_{3}(x)-Q_{3}(x)\right|
\end{aligned}
$$

$P$ is a protein for which the significant spot is to be predicted, and $Q$ is a protein to be compared with $P . P_{1}(x)$, $Q_{1}(x)$ are the values of the angle histogram, $P_{2}(x)$, $Q_{2}(x)$ are the values of the height feature vector, and $P_{3}(x)$, $Q_{3}(x)$ are the values of the physical property histogram. Then, at all feature points, it is assumed that the sum of all feature values is normalized to $N$ as follows.

$$
\begin{aligned}
w_{1} \sum_{x=1}^{m * l} P_{1}(x) & +w_{2} \sum_{x=1}^{l} P_{2}^{\prime}(x) \\
& +w_{3} \sum_{x=1}^{c * d * e} P_{3}(x)=N \\
w_{1} \sum_{x=1}^{m * l} Q_{1}(x) & +w_{2} \sum_{x=1}^{l} Q_{2}^{\prime}(x) \\
& +w_{3} \sum_{x=1}^{c * d * e} Q_{3}(x)=N
\end{aligned}
$$

Also, $P_{2}^{\prime}(x)$ and $Q_{2}^{\prime}(x)$ are expressed by the following equations.

$$
\begin{aligned}
& P_{2}^{\prime}(x)=1+\frac{P_{2}(x)}{\operatorname{dis}(x)} \\
& Q_{2}^{\prime}(x)=1+\frac{Q_{2}(x)}{\operatorname{dis}(x)}
\end{aligned}
$$

$\operatorname{dis}(x)$ is the average distance between feature points and nearby points in bin $x$ of distances. As a result, $P_{2}^{\prime}(x)$ and $Q_{2}^{\prime}(x)$ always take values from 0 to 2.0. At this time, if $S$ is above a certain threshold, the feature point pairs can be considered similar.

\subsection{Prediction of significant spot with biclustering}

For many pockets that bind similar ligands, when a set of similar feature points is found, the feature points can be considered to correspond to sites that play an important role in binding to that ligand. Therefore, for a set of feature points that constitute a protein pocket, a set of feature points similar to them is extracted from many pockets, and biclustering processing is performed to realize extraction of the significant spot. As a method of biclustering to predict the significant spot, we use BISERS ${ }^{(12)}$ proposed by Nishimura et al.

\section{Experiment and evaluation}




\subsection{Experiment setting}

60 types of proteins are used in the evaluation experiment. Each protein is already known to bind to any of 15 types of ligands. These proteins are a data set in which four binding proteins are prepared for one ligand. The analyzed proteins are comprehensively matched with all other proteins in the data set. A total of $60 \times 59$ matching processes compare 60 types of proteins with other proteins and perform biclustering processing using the results. In order to show the effectiveness of the proposed method, we compared it with the method of Nishimura et al. ${ }^{(12)}$. In the method of Nishimura et al., feature points are extracted by a curvature-based approach ${ }^{(10)}$, and comparison is performed between feature points using point feature histograms ${ }^{(13)}$ as feature quantities. In this method, in order to create feature points consisting a pocket, information on nearby points at a certain distance is referenced. In addition, two histograms of physical property and position are used as feature value. From this, the method of Nishimura et al. is called "Fixed Extraction with 2 histogram". (described as "FE2") Moreover, we compare with a method in which a pocket is extracted based on positional information of the ligand and the two histograms used in the method of Nishimura et al are introduced as feature value. We call this method "Ligand Based Extraction with 2 histogram"(described as "LBE2") The objective for this comparison is to investigate which of the pocket extraction method and the feature value generation method greatly affects the result. We perform the same experiment using these three methods and evaluate each significant spot. The parameters of the proposed method are set as follows.

- Cutting distance for pocket extraction dis = $7.5 \AA$.

- Parameters of angle histogram $m=5, l=10$.

- Parameters of height feature vector $l=10$.

- Parameters of physical property histogram $c=$ $4, d=3, e=5$.

- Weight parameter at matching $w_{1}=1, w_{2}=$ $5, w_{3}=1$.

- Threshold of similarity when matching $S=0.60$.

- Reference value for normalization at matching $N=1.0$.

We use the information of residues recorded in PDB to be involved in binding as an evaluation of significant spot prediction. Residues involved in binding are residues in which atoms other than the constituent atoms of the protein or nucleic acid called "HETATM" are present within $5 \AA$,

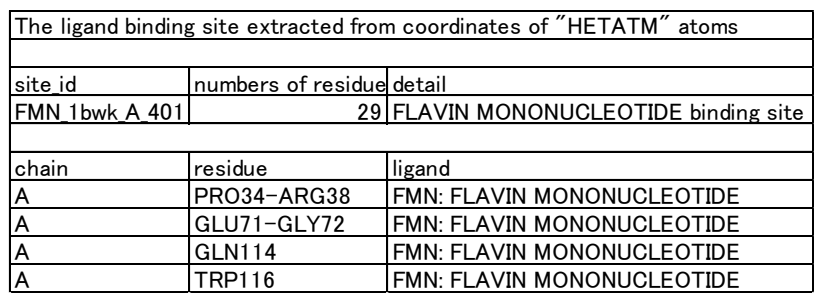

Fig. 6. Ligand binding site "HETATM" data(12).

Table 1. Experimental result.

\begin{tabular}{|c|c|}
\hline & average precision \\
\hline FE2 & 0.701 \\
\hline LBE2 & 0.657 \\
\hline proposed method & 0.705 \\
\hline
\end{tabular}

and are recorded as shown in Fig. 6. Let this be the ground truth. Then, for each feature point that constitutes the significant spot, if the residue spatially closest to that point is the above-mentioned "residue involved in binding", it is judged that the feature point is correctly extracted. Among the feature points that constitute the significant spot, the ratio (precision) of the correctly extracted feature points is calculated, and the method is evaluated.

\subsection{Experimental result and evaluation}

From the experimental results, we compare two comparison methods and the proposed method. In order to evaluate each method, the average value of precision is shown in Table 1.

As shown in this table, the average precision of LBE2 was slightly lower than FE2. This may be caused by changing the pocket extraction method. However, for the mean value of precision, few significant differences were found between FE2 and the proposed method. From this, it is thought that changing the feature value generation method has a great effect on the results. For a more detailed discussion, Fig. 7 shows a scatter plot with the number of points predicted as significant spot on the horizontal axis and the precision on the vertical axis.

In FE2, the number of feature points extracted is extremely small, and a very small part of a large amount of correct data is correctly extracted. This is the reason why the value of precision is high in FE2. On the other hand, the proposed method extracts feature points whose amount is much larger than FE2 as significant spot. From this, it can be considered that the proposed method shows better results than FE2 in the prediction of the significant spot.

In LBE2, more feature points than FE2 are extracted as significant spot. However, compared with the proposed 


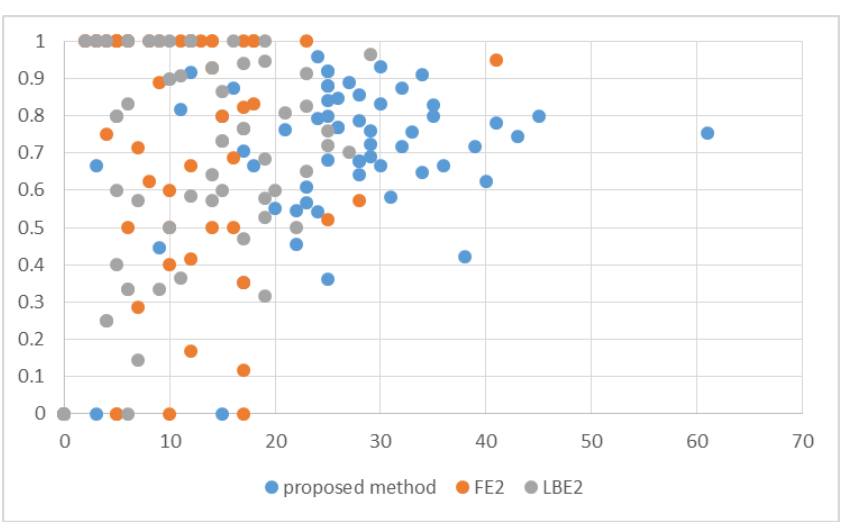

Fig. 7. Precision and number of feature points predicted as significant spot.

Table 2. Precision and number of feature points predicted as significant spot in protein 3ign-A.

\begin{tabular}{|c|c|c|}
\hline & feature points & precision \\
\hline FE2 & 0 & 0.0 \\
\hline LBE2 & 3 & 1.0 \\
\hline proposed method & 25 & 0.84 \\
\hline
\end{tabular}

method, the number of feature points extracted by LBE2 is small. From this, it can be interpreted that the feature value generation method has a great influence on the number of the feature points extracted as the significant spot. However, as shown in Table 2, although the significant spot could not be extracted in FE2, there was also protein that could be extracted in LBE2. From this, it is considered that the pocket extraction method also has some influence.

On the other hand, some proteins with extremely low precision exist in the results of the proposed method. The cause is that the binding site on the ligand side is different. As an example, Fig. 8 and 9 show two different proteins that bind to the same ligand. The two have different surface properties, as is obvious from the figure. In each pocket, even if they bind to the same ligand, they do not necessarily bind in the same plane. Therefore, in order to further improve the accuracy, biclustering needs to be performed separately according to the binding site on the ligand among the proteins binding to the same ligand. Similarly, analysis of the binding site on the ligand is also essential.

\section{Conclusion}

In this paper, we proposed a method to predict the significant spot about binding between protein and ligand by extracting feature value considering the surface information of protein and comparing feature points. In the proposed method, positional feature value using normal vector and position coordinates and feature value about

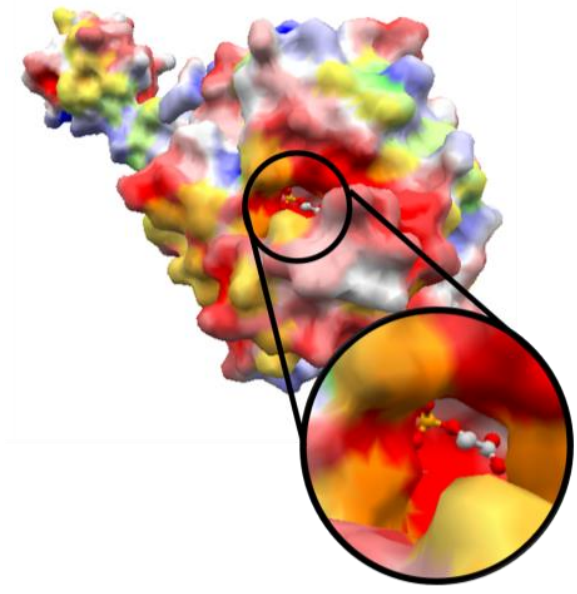

Fig. 8. Protein 1xuz-A.

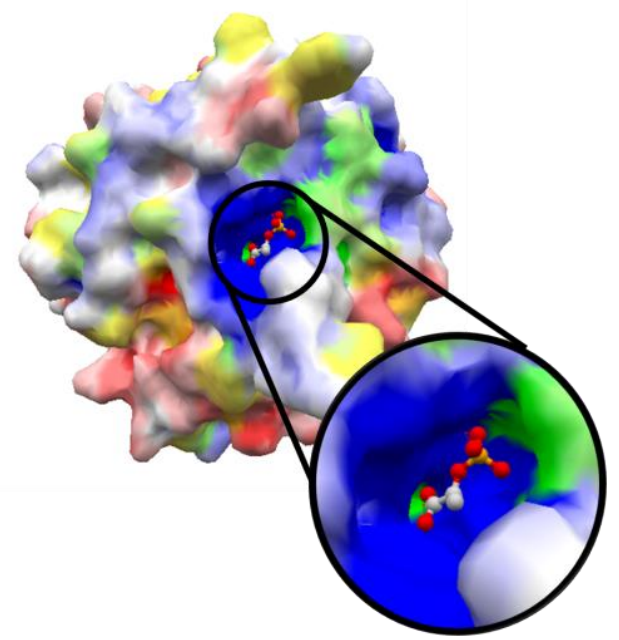

Fig. 9. Protein 3fyo-D.

physical properties using hydrophobicity of amino acids and electrostatic potential of molecular surface data are created. At that time, by classifying these by distance, it is possible to separate and hold the property that changes according to the distance from the feature point, and it is possible to compare more detailed information. In order to show the effectiveness of the proposed method, extraction experiments of significant spots were conducted. As a result, it was possible to predict a feature point about twice as large as that of comparison method as significant spot while maintaining the value of precision. The result shows that the proposed method can extract more significant spots with higher accuracy than the comparison method. As a problem in the proposed method, the pocket of each protein binding to the same ligand may not be considered even if the binding state or binding site of the ligand is different. This can't be grasped by the information only on the protein surface, so in the future it will be necessary to analyze the structure and properties of the ligand side. 


\section{References}

(1) C.I. Branden, J. Tooze : "Introduction to Protein Structure", Garland Science, 1999

(2) J.C. Setubal, J. Meidanis : "Introduction to Computational Molecular Biology”, Pws Pub Co, 1997

(3) J. Jiménez, M. Škalič, G. Martínez-Rosell, G.D. Fabritiis : " $\mathrm{K}_{\mathrm{DEEP}}$ : Protein-Ligand Absolute Binding Affinity Prediction via 3D-Convolutional Neural Networks", J. Chem. Inf. Model., Vol.58, Issue 2, pp.287-296, 2018

(4) T. Krotzky, T. Rickmeyer, T. Fober, G. Klebe : " Extraction of Protein Binding Pockets in Close Neighborhood of Bound Ligands Makes Comparisons Simple Due to Inherent Shape Similarity", J. Chem. Inf. Model., Vol.54, Issue 11, pp.3229-3237, 2014

(5) A. Kumar, K.Y. Zhang : "Hierarchical virtual screening approaches in small molecule drug discovery", Methods, Vol.71, pp.26-37, 2015

(6) M. Tsubaki, K. Tomii, J. Sese : "Compound-protein interaction prediction with end-to-end learning of neural networks for graphs and sequences", Bioinformatics, Vol.35, Issue 2, pp.309-318, 2019

(7) B. Yao, Z. Li, M. Ding, M. Chen :" Three-dimensional protein model similarity analysis based on salient shape index”, BMC Bioinformatics, Vol.17, 131, 2016

(8) K. Kinoshita, H. Nakamura : "Identification of Protein Molecular Functions from eF-site, a Molecular Surface Database", Biophysics and Physicobiology, Vol.42, No. 1, pp. 20-23, 2002

(9) H. M. Berman, J.Westbrook, Z. Feng, G. Gilliland, T.N. Bhat, H. Weissig, I. N. Shindyalov, P. E. Bourne : "The Protein Data Bank", Nucleic Acids Research, Vol.28, pp.235-242, 2000

(10) H. T. Ho, D. Gibbins : "A Curvature-based Approach for Multi-scale Feature Extraction from 3D Meshes and Unstructured Point Clouds", IET Computer Vision, Vol.3, No.4, pp.201-212, 2009

(11) J.Kyte, R.F.Doolittle : "A simple method for displaying the hydropathic character of a protein", Journal of Molecular Biology, Vol.157, Issue 1, pp.105-132, 1982

(12)H. Nishimura, T. Ohkawa : "A New Biclustering Algorithm with Exclusive Random Selection of Columns for Predicting Recognition Spots on Protein Molecular Surfaces", International Journal of Bioscience, Biochemistry and Bioinformatics, Vol. 8, No.1, pp.11-19, 2018

(13) R. B. Rusu, N. Blodow, Z. C. Marton, M. Beetz :
"Aligning point cloud views using persistent feature histograms", Proceedings of IEEE/RSJ International Conference on Intelligent Robots and Systems, pp.3384-3391, 2008 